\title{
Hypothermia for neonatal hypoxic-ischemic encephalopathy: may an early amplitude-integrated EEG improve the selection of candidates for cooling?
}

\author{
Luca Filippi ${ }^{1}$, Serena Catarzi ${ }^{1}$, Elena Gozzini ${ }^{1}$, Patrizio Fiorini ${ }^{1}$, Melania Falchi ${ }^{2}$, Tiziana Pisano ${ }^{2}$, \\ Giancarlo la Marca², Gianpaolo Donzelli ${ }^{3}$ \& Renzo Guerrini ${ }^{2}$
}

${ }^{1}$ Neonatal Intensive Care Unit, Medical Surgical Feto-Neonatal Department, ${ }^{2}$ Department of Neurology and Neurosurgery, "A. Meyer" University Children's Hospital, Florence, Italy, and ${ }^{3}$ Department of Sciences for Woman and Child's Health, University of Florence, Meyer Children's Hospital Florence, Italy

Objective: To report our experience in the selection of newborns candidate to therapeutic hypothermia. Methods: Retrospective study involving 47 newborns suffering from perinatal asphyxia from January 2008 to September 2011. Results: Thirty-five of 47 newborns admitted to our hospital fulfilled metabolic and neurological criteria for recruitment and were cooled. aEEG was carried out in $\mathbf{2 6}$ of them and resulted always abnormal. In three of the 12 newborns with only metabolic criteria, aEEG was moderately abnormal. They were cooled and their outcome (evaluated by General Movements and Griffiths Mental Development Scales for children aged $0-2$ years) is good. Three additional newborns who only met the metabolic criterion reached our hospital after the therapeutic window for hypothermia and exhibited seizures; their outcome is poor. Conclusions: In our experience, the inclusion of aEEG in the entry criteria would not have precluded newborns with neurological criteria from cooling. On the contrary, without an early aEEG, we would have excluded from hypothermia infants with moderate hypoxic-ischemic encephalopathy without precocious neurological signs who exhibited only the metabolic criterion, but with abnormal aEEG. If further studies will confirm that early aEEG might identify newborns suitable for cooling even in the absence of clinical signs, a revision of the entry criteria should be considered.

Keywords: asphyxia, hypoxic-ischemic encephalopathy, neuroprotection, newborn

Abbreviations: aEEG: amplitude integrated EEG; Cho: choline; Cr: creatine phosphocreatine; DQ: developmental quotient; GMDS: Griffiths Mental Development Scales; GMs: general movements; HIE: hypoxic-ischemic encephalopathy; MRI: magnetic resonance imaging; NAA: N-acetylaspartate; NICU: neonatal intensive care unit; SD: standard deviations

\section{Introduction}

Perinatal asphyxia complicated by moderate or severe hypoxicischemic encephalopathy (HIE) is one of the major causes for neonatal death or later neurodevelopmental sequelae [1]. Its incidence is estimated to be 3-9 cases out of 1000 live births [2]. About $10 \%$ of newborns with moderate HIE die and about $30 \%$ of those who survive have neurological impairment. Such percentages raise in infants with severe encephalopathy to around 60 and $100 \%[3,4]$. Selective head cooling or whole-body cooling to a target core temperature of $33.5^{\circ} \mathrm{C}$ for around $72 \mathrm{~h}$, to be started within $6 \mathrm{~h}$ after birth (the therapeutic window for potential neurological rescue) [5], is reported to significantly improve newborns' survival and to reduce neurological impairment [6-9]. It is therefore essential to identify the candidates for this treatment as soon as possible [10].

The most used entry criteria include a metabolic indication of hypoxic-ischemic injury (low Apgar scores and/or persisting need for resuscitation and/or severe acidosis) and at least two or three clinical neurological findings of neonatal encephalopathy (altered state of consciousness, abnormalities of tone, reflexes, posturing, autonomic dysfunction and seizures) [5-8], corresponding to Sarnat grade 2-3 [11].

The amplitude integrated electroencephalography (aEEG) has been adopted as entry criteria for the treatment with hypothermia only in a few of the major trials $[6,9,12]$, where the newborns treated only after all three major criteria (metabolic, neurological and electroencephalographic) were sequentially fulfilled. The role of aEEG in selecting newborns for cooling remains controversial because of its low predictive value and the risk of reserving hypothermia only to newborns with more severe asphyxia [13]. However, no study has explored the possibility that aEEG could identify newborns with anomalous electrographic activity, typical of a moderate encephalopathy, in the absence of neurological signs, who, according to standard inclusion criteria, would not be cooled.

In this paper, we report our experience with asphyxiated newborns hospitalized in our Neonatal Intensive Care Unit (NICU), where some newborns admitted with metabolic criterion

Correspondence: Luca Filippi M.D., Neonatal Intensive Care Unit, Department of Critical Care Medicine, “A. Meyer” University Children’s Hospital, viale Pieraccini, 24 I-50139 Florence, Italy. Tel: ++39-(0)55-5662434. Fax: ++39-(0)55-5662400. E-mail: 1.filippi@meyer.it 
only exhibited abnormal aEEG. This experience seems to suggest that aEEG could be necessary for selecting neonates with HIE who would benefit from hypothermia.

In view of this specific experience, we also discuss the potential role of an early aEEG evaluation in the identification of candidates for the treatment with hypothermia.

\section{Patients and methods}

We performed a retrospective cohort study identifying from medical records all term newborns (gestational age 37-42 weeks) admitted to the 16 beds NICU of the A. Meyer University Children's' Hospital, for perinatal asphyxia, from January 2008 to September 2011.

The treatment with therapeutic hypothermia was initially reserved to newborns with gestational age $\geq 36$ weeks and birth weight $\geq 1800 \mathrm{~g}$ who fulfilled the following two criteria modified from Sarnat and Sarnat [11]: (i) metabolic criteria: Apgar score $\leq 5$ at $10 \mathrm{~min}$, or persisting need for resuscitation, including endotracheal intubation or mask ventilation for more than 10 min after birth, or acidosis ( $\mathrm{pH} \leq 7.0$ and/or base deficit $\geq-16$ $\mathrm{mmol} / \mathrm{L}$ in umbilical cord blood or arterial, venous or capillary blood) within $60 \mathrm{~min}$ from birth; (ii) neurological criteria: moderate to severe encephalopathy consisting of altered state of consciousness (irritability, lethargy, stupor or coma) and $\geq 1$ of the following signs: hypotonia, or abnormal reflexes including oculomotor or pupil abnormalities, or absent or weak suctioning, or clinical seizures. Newborns older than $6 \mathrm{~h}$ of age at the time of evaluation and newborns with major congenital abnormalities, congenital viral infections or evidence encephalopathy other than HIE were excluded. Outborn newborns who met both the above criteria were initially cooled to around $35^{\circ} \mathrm{C}$ at their birth hospital, by stopping the heating and applying ice packs close to their head and body during the transfer to NICU. Upon arrival, newborns were evaluated again by skilled and qualified neonatologists to evaluate their suitability for therapeutic hypothermia. In the presence of both criteria, newborns were cooled to $33.5^{\circ}$ using a cooling blanket (Blanketrol ${ }^{\circledR}$ III, Hyper-hypothermia System, Cincinnati Sub-Zero, Cincinnati, OH, USA), connected by a servomechanism with an esophageal probe.

After $72 \mathrm{~h}$ of hypothermia, newborns were gradually rewarmed to $36.5-37^{\circ} \mathrm{C}$ by increasing the automatic control set point of $0.5^{\circ} \mathrm{C} / \mathrm{h}$. Newborns who fulfilled both entry criteria were enrolled until August 2009 in a pilot study that evaluated the safety of oral topiramate administration associated to hypothermia [14] and those admitted from February 2010 in a randomized controlled study evaluating the efficacy of combination treatment with topiramate and hypothermia [15].

From November 2008, all newborns referred to our NICU for perinatal asphyxia underwent aEEG recording (BRM 3®, BrainZ Instruments, Auckland, New Zealand) more than 20 min, starting as soon as they were admitted. The needle electrodes were placed bilaterally at the central and parietal positions (according to the $\mathrm{C} 3$ and P3 on the left and $\mathrm{C} 4$ and P4 on the right placement on the newborn infant). The definition "moderately abnormal" indicates a background tracing with upper margin $>10 \mu \mathrm{V}$ and lower margin $\leq 5 \mu \mathrm{V}$; "severely abnormal" pattern refers to a tracing with upper margin $<10 \mu \mathrm{V}$ and lower margin $<5 \mu \mathrm{V}$; often, this is accompanied by bursts of high voltage activity ("burst suppression") [16]. Seizures were identified as periods of sudden increase in voltage, accompanied by a narrowing of the band of aEEG activity followed by a brief period of suppression [16] or by a build-up of rhythmic activity of increasing amplitude and decreasing frequency. The aEEG recordings obtained during the evaluation for the potential cooling were subsequently evaluated again for abnormalities by three of the investigators (FM, TP and GR) certified to read the aEEG recordings. An abnormal aEEG was followed by standard EEG. Both studies were approved by the Institutional Review Board, and informed consent was obtained from the children's parents. While for newborns enrolled in the prospective trial, a complex neurodevelopmental and neuroradiologic follow-up was planned; for those who fulfilled only the first metabolic criterion, neurodevelopment was evaluated at 3 months of life with the General Movements (GMs) [17] and Griffiths Mental Development Scales (GMDS) for children aged 0-2 years [18]. The total developmental quotient (DQ) and subquotients were calculated. The normal DQ is usually evaluated to be $100 \pm 13$. All infants were assessed by neuropsychiatrists specialized in neurodevelopmental examination and who had completed an accredited training course on the GMDS (FM, TP and GR). In newborns with abnormal aEEG, a cerebral magnetic resonance imaging (MRI) was planned within the first week of life and then at the 3rd and 18th month. MRI of the brain was performed using a $1.5 \mathrm{~T}$ magnet with $\mathrm{T} 1$ and $\mathrm{T} 2$ weighted imaging sequences in the coronal, axial and sagittal planes.

An adverse outcome was defined by an absence of fidgety movements at 3 months at the GMs, a DQ $<2$ standard deviations (SD) at GMDS and the presence of brain lesions characteristic of HIE at MRI (involvement in the basal ganglia and thalamus,

Table I. Demographic data and outcome of newborns treated with hypothermia.

\begin{tabular}{|c|c|}
\hline Newborns treated with hypothermia, $n=35$ & \\
\hline Male, $n(\%)$ & $23(65.7)$ \\
\hline Gestational age, weeks, mean $\pm \mathrm{SD}^{\mathrm{a}}$ & $38.6 \pm 2.6$ \\
\hline Birth weight, g, mean \pm SD & $3168 \pm 545$ \\
\hline Cesarean section, $n(\%)$ & $21(60.0 \%)$ \\
\hline Stained amniotic fluid, n (\%) & $17(48.6 \%)$ \\
\hline Outborn, n (\%) & $35(100.0 \%)$ \\
\hline Apgar Score, 1 min, mean \pm SD & $2.0 \pm 2.3$ \\
\hline Apgar Score, 5 min, mean \pm SD & $3.9 \pm 2.3$ \\
\hline Crib score, mean $\pm \mathrm{SD}$ & $4.1 \pm 2.0$ \\
\hline Arterial pH, mean \pm SD & $6.855 \pm 0.17$ \\
\hline Arterial $\mathrm{BE}, \mathrm{mEq} / \mathrm{L}$, mean $\pm \mathrm{SD}$ & $-21.6 \pm 7.2$ \\
\hline Arterial lactic acid, mmol/l, mean \pm SD & $16.1 \pm 4.4$ \\
\hline Hour hypothermia beginning, mean \pm SD & $3.6 \pm 1.3$ \\
\hline Survival rate at discharge, $n(\%)$ & $30(85.7)$ \\
\hline Cerebral magnetic resonance at discharge, $n(\%)$ & $34(97.1)$ \\
\hline Normal, $n(\%)$ & $13(38.2)$ \\
\hline WM isolated, $n(\%)$ & $1(2.9)$ \\
\hline $\mathrm{WM}+\mathrm{COR}, n(\%)$ & 0 \\
\hline BGT isolated, $\mathrm{n}(\%)$ & $12(35.3)$ \\
\hline $\mathrm{BGT}+\mathrm{WM}, \mathrm{n}(\%)$ & $2(5.9)$ \\
\hline $\mathrm{BGT}+\mathrm{COR}, \mathrm{n}(\%)$ & $1(2.9)$ \\
\hline BGT+COR+WM, n (\%) & $5(14.7)$ \\
\hline Neurological evaluation at follow-up, $n(\%)$ & $34(97.1 \%)$ \\
\hline Normal & $19(55.9)$ \\
\hline Cerebral palsy and developmental delay $n(\%)$ & $4(11.8)$ \\
\hline Cerebral palsy, developmental delay and epilepsy, $n(\%)$ & $6(17.6)$ \\
\hline Deceased & $5(14.7)$ \\
\hline Not available & $1(2.9 \%)$ \\
\hline
\end{tabular}

${ }^{a}$ Calculated from maternal menstrual history, obstetrical data or by Ballard's score. $\mathrm{BE}$, base excess; BGT, basal ganglia/thalamus; COR, cortex; CRIB, Clinical Risk Index for Babies; SD, standard deviation; WM, white matter. 


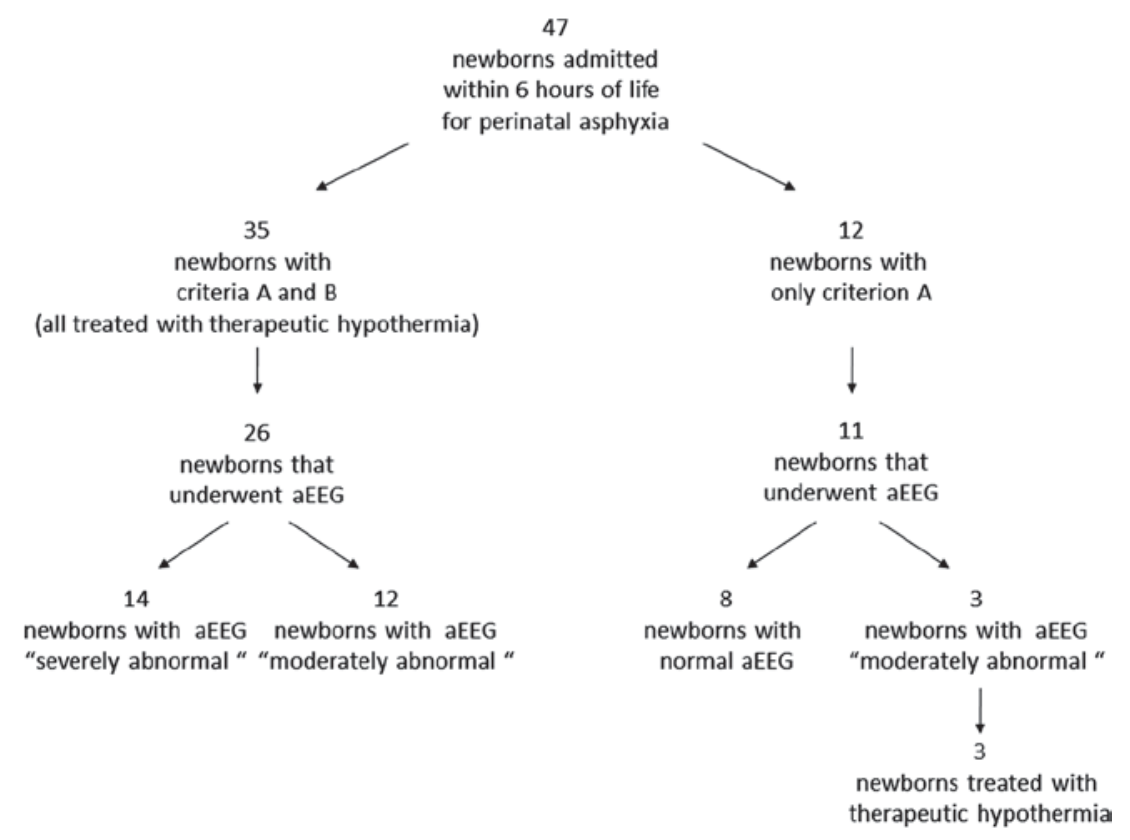

Figure 1. Patients arrived to our NICU for perinatal asphyxia.

posterior limb of the internal capsule, subcortical white matter and cortical gray matter).

\section{Results}

During the period of the study, 47 asphyxiated newborns were admitted in our NICU. Thirty-five met both admission criteria and were cooled. Demographic data and neurologic outcome of these newborns are shown in Table I. aEEG recording was started immediately after their admission in 26 of these newborns, at a median age of $3.4 \pm 1.1 \mathrm{~h}$ after birth. A moderately abnormal aEEG was detected in 12 newborns (46.2\%) and a severely abnormal aEEG in 14 (53.8\%) (Figure 1). The remaining nine newborns were evaluated with a standard EEG that was abnormal in all.

Twelve additional newborns who only exhibited the metabolic criteria were transferred within the first $6 \mathrm{~h}$ of life to our NICU to evaluate their suitability for the treatment with therapeutic hypothermia. Two of these neonates exhibited mild respiratory distress in the first hours of life, but none required mechanical ventilation; none of these infants received sedation, and a detailed clinical evaluation confirmed the absence of neurological criterion in all the newborns. Eleven newborns were subjected to aEEG. Eight of them exhibited a normal EEG pattern and their neurodevelopmental outcome was normal. Three exhibited a moderately abnormal EEG pattern and their outcome is discussed in the following section. Demographic data of these three patients are shown in Table II (patients 1, 2 and 3).

At the arrival in NICU, patient 1 was hyperalert and his aEEG is shown in Figure 2. A standard EEG revealed a low voltage path. As this electrophysiological finding was consistent with a Sarnat grade 2, we decided to cool him. At $12 \mathrm{~h}$, seizures occurred that were treated with phenobarbital. Cerebral ultrasounds at 4 days of life revealed mild diffuse parenchymal hyperechogenicity; MRI at 5 days was normal; the spectroscopy study showed a reduction in the ratio $\mathrm{N}$-acetilaspartate (NAA)/creatine phosphocreatine $(\mathrm{Cr}$ ), without lactic acid. GMs were normal at 3 months (presence of fidgety) and his DQ at the actual age is in the normal range.
Patient 2 showed in the first hours of life only overactive stretch reflexes; her aEEG is shown in Figure 2. A standard EEG showed a low voltage path with alternating suppression and anterior non-reactive irregular activity. As a result of this observation, therapeutic hypothermia was started. Cerebral ultrasound at 3 days showed mild periventricular hyperechogenicity. Brain MRI at 5 days showed increased signal intensity in the basal ganglia and in the posterior left semioval center. Spectroscopy showed a reduction in the pool of NAA, a slight increase in choline (Cho)/Cr with lactic acid in the basal ganglia and left semioval center. At 3 months, she showed normal GMs, her DQ at the actual age is in the normal range and her MRI is actually normal.

Patient 3 was cooled because aEEG was moderately abnormal (Figure 2), and standard EEG showed a low voltage and asymmetric path. At $16 \mathrm{~h}$, seizures required treatment with phenobarbital. At her 5th day of life brain, MRI spectroscopy revealed lactate in the white matter and in the basal ganglia. Fidgety was present at 3 months, her DQ at actual age is in the normal range and cerebral MRI is normal.

In the same period, three additional asphyxiated newborns were admitted suffering from seizures that had developed after the therapeutic window for treatment with hypothermia had passed. At the birth hospital, these newborns had been considered non suitable for hypothermia because clinical evaluation, carried out by not-trained personnel, had failed to detect neurological signs of HIE, neither aEEG had been performed. These patients are presented in Table II as patients 4, 5 and 6.

Patient 4 was resuscitated at birth, but clinical examination was normal in the first hours of life. At $20 \mathrm{~h}$, clonic seizures appeared in the lower limbs. EEG showed a series of electrical seizures and therapy with phenobarbital was started. At 24 hours of life, she was transferred to our NICU. Standard EEG at $28 \mathrm{~h}$ showed paroxysmal abnormalities. Intravenous phenytoin was added and in the eighth day of life EEG improved. Brain MRI at 3 days showed a diffuse subcortical white matter and basal ganglia hyperintensity. At 3 months, GMs were pathological, with absence of fidgety and poor repertoire. Nowadays, he has a pathological DQ $(2>\mathrm{SD}<3)$ and his cerebral MRI is abnormal. 
Table II. Demographic and clinical data of the reported newborns.

\begin{tabular}{|c|c|c|c|c|c|c|}
\hline Patient & 1 & 2 & 3 & 4 & 5 & 6 \\
\hline Sex & $\mathrm{M}$ & $\mathrm{F}$ & F & $\mathrm{M}$ & $\mathrm{F}$ & $\mathrm{M}$ \\
\hline $\begin{array}{l}\text { Gestational } \\
\text { age, weeks }\end{array}$ & $41+1$ & $39+2$ & 38 & 38 & $41+2$ & $42+2$ \\
\hline Birth weight, g & 3690 & 3350 & 3000 & 2565 & 2500 & 2890 \\
\hline $\begin{array}{l}\text { Type of } \\
\text { delivery }\end{array}$ & $\mathrm{Sp}$ & $\mathrm{Sp}$ & $\mathrm{EC}$ & $\mathrm{Sp}$ & $\mathrm{EC}$ & $\mathrm{Sp}$ \\
\hline $\begin{array}{l}\text { Obstetric risk } \\
\text { factor }\end{array}$ & SAF & $\begin{array}{l}\text { VE and } \\
\text { SAF }\end{array}$ & $\begin{array}{l}\text { PA and } \\
\text { HRD }\end{array}$ & $\mathrm{VE}$ & SAF & HRD \\
\hline $\begin{array}{l}\text { Intubation in } \\
\text { delivery room }\end{array}$ & yes & yes & no & yes & no & No \\
\hline $\begin{array}{l}\text { Apgar Score, } \\
1 \mathrm{~min}\end{array}$ & 3 & 5 & 3 & 4 & 6 & 3 \\
\hline $\begin{array}{l}\text { Apgar Score, } \\
5 \mathrm{~min}\end{array}$ & 5 & 5 & 6 & 4 & 6 & 8 \\
\hline $\begin{array}{l}\text { Arterial pH, } \\
-\log \left(\mathrm{H}^{+}\right)\end{array}$ & 6.82 & 6.88 & 6.84 & 6.84 & 6.87 & 7.09 \\
\hline $\mathrm{pCO}_{2}, \mathrm{mmHg}$ & 93.2 & 72.3 & 77.3 & 24.5 & 56.6 & 64 \\
\hline $\mathrm{pO}_{2}, \mathrm{mmHg}$ & 47.5 & 12.3 & 97.2 & 56.7 & 45.8 & 25.1 \\
\hline $\begin{array}{l}\text { Arterial BE, } \\
\mathrm{mEq} / \mathrm{L}\end{array}$ & -28.3 & -20.3 & -19.1 & -25.9 & -24.4 & -16.8 \\
\hline $\begin{array}{l}\text { Arterial } \\
\mathrm{HCO}_{3} \\
\mathrm{mmol}^{\prime} \mathrm{L}\end{array}$ & 5.0 & 7.2 & 7.4 & 6.0 & 7.8 & 12.2 \\
\hline $\begin{array}{l}\text { Arterial lactic } \\
\text { acid, } \mathrm{mmol} / \mathrm{L}\end{array}$ & 17.6 & 11.9 & 17.4 & 12.1 & 10.9 & 11.2 \\
\hline $\begin{array}{l}\text { Clinical } \\
\text { Seizures } \\
\text { (hours of life) }\end{array}$ & 12 & - & 16 & 20 & 16 & 10 \\
\hline Phenobarbital & Yes & - & Yes & Yes & Yes & Yes \\
\hline $\begin{array}{l}\text { Therapeutic } \\
\text { hypothermia }\end{array}$ & Cooled & Cooled & Cooled & Not cooleds & Not cooled & $\begin{array}{l}\text { d Not } \\
\text { cooled }\end{array}$ \\
\hline $\begin{array}{l}\text { Actual age } \\
\text { (months) }\end{array}$ & 18 & 18 & 9 & 26 & 6 & 1 \\
\hline $\begin{array}{l}\text { Fidgety at } 3 \\
\text { months }\end{array}$ & present & present & present & absent & absent & N/A \\
\hline $\begin{array}{l}\mathrm{DQ} \text { at GMDS } \\
\text { (SD) }\end{array}$ & normal & normal & normal & $>2 \mathrm{SD}<3$ & $<3 \mathrm{SD}$ & N/A \\
\hline $\begin{array}{l}\mathrm{HC} \text { (centile) at } \\
\text { actual age }\end{array}$ & $50^{\circ}$ & $50-75^{\circ}$ & $25-50^{\circ}$ & $<3^{\circ}$ & $<3^{\circ}$ & $25-50^{\circ}$ \\
\hline
\end{tabular}

DQ, developmental quotient; VE, vacuum extractor; EC, emergency cesarean; GMDS, Griffiths Mental Development Scales; HC, head circumference; HRD, heart rate deceleration; PA, placental abruption; SAF, stained amniotic fluid; Sp, spontaneous.

Patient 5 was neurologically normal in the first hours, but she arrived to our NICU at $16 \mathrm{~h}$ manifesting seizures that were treated with phenobarbital and midazolam. EEG showed abnormalities in both central regions and paroxysmal abnormalities that were unresponsive to pyridoxine and phenytoin, but were attenuated by high doses of midazolam. Brain MRI at 12 days showed bilateral frontal and posterior ischemic lesions and widespread reduction in the subcortical white matter. Fidgety at 3 months was absent and her cerebral MRI is still pathological. Her DQ at actual age is out of the normal range $(\mathrm{SD}<3)$.

Patient 6 was resuscitated at birth, but appeared neurologically normal in the first hours of life. Seizures, appeared at $10 \mathrm{~h}$ of life, were successfully treated with midazolam after failure of phenobarbital and phenytoin. EEG showed a discontinuous tracing with burst suppression. Brain MRI at 5 days showed frontal, temporal, occipital, thalamic ischemic lesions and widespread reduction in the subcortical white matter.

\section{Discussion}

Therapeutic hypothermia is currently the most effective neuroprotective strategy for newborns with HIE $[6-9,19]$. The CoolCap Trial demonstrated that cooling was beneficial in the subgroup of newborns with moderate aEEG abnormalities [6], typical of Sarnat grade 2 [11]. On the contrary, when newborns exhibited severe clinical encephalopathy or severely impaired background activity on early aEEG, outcome was still likely to be extremely poor [20$22]$, despite hypothermia [5,21]. Therefore, it is essential to identify the largest number of newborns with a moderate encephalopathy.

Although EEG is useful to identify suitable newborns for hypothermia due to its capability to distinguish newborns with Sarnat grade 2 from newborns with Sarnat 1 [11], its use in this context remains controversial. Several studies have demonstrated that aEEG is easier to be interpreted than standard EEG, but equally accurate and reproducible [23]. It correlates well with neurodevelopmental outcome of full-term infants with HIE $[13,24,25]$, and it is considered the best single predictor of neurologic outcome [26]. In some studies aEEG was more specific and exhibited a higher positive predictive value when compared with the neurologic examination performed in the first hours after delivery [13]. This finding, together with the observation that moderate hypothermia does not affect aEEG amplitude [27], could advocate its use in newborns who are at risk of HIE for selecting suitable candidates for cooling. This approach was adopted in the CoolCap study [6], the TOBY Trial [9] and the NeonEuro study [12], where aEEG was employed as a third entry criterion, in addition to metabolic and neurological parameters. However, in other studies this strategy was considered to be misleading because, according to these criteria, mainly the sickest newborns suffering from a more serious HIE would be selected [28]. The same question has been addressed by Sarkar et al. who found a normal or a non-qualifying aEEG in a few newborns with metabolic and clinical signs of severe encephalopathy [29]. These newborns were not cooled, but a significant part of them showed clinical signs and neuroradiologic lesions with severe HIE afterwards. According to these authors, aEEG was judged not advisable as additional selection criterion because of the low negative predictive value in the first hours of life, and other important trials did not consider aEEG as a criterion to select newborns to be treated with hypothermia $[6,7]$.

In our experience, all patients who fulfilled both metabolic and neurological criteria exhibited an abnormal aEEG. Therefore, in contrast with the mentioned study, should we have used aEEG as entry criteria, we would not have precluded newborns with neurological signs of HIE from cooling. On the contrary, without an early aEEG, we would have excluded from treatment with hypothermia infants exhibiting the metabolic criterion only who, however, subsequently demonstrated neurological disorders or abnormal aEEG.

Recently, it has been demonstrated that the aEEG is influenced by electrode location and interelectrode space. The amplitude increased linearly and significantly with the interelectrode distances [30]. This means that if the electrodes are spaced close to each other, the trace could be often outside the voltage normal criteria. However, all the reported cases with moderately abnormal aEEG also had significantly abnormal EEG trace.

Mild aEEG anomalies were observed in $46.2 \%$ of newborns in our study, whereas severe anomalies were recorded in $53.8 \%$ of the cases. These percentages are rather different from those observed in the CoolCap study, where most newborns had moderate EEG anomalies [6], but are similar to those described in the TOBY trial 


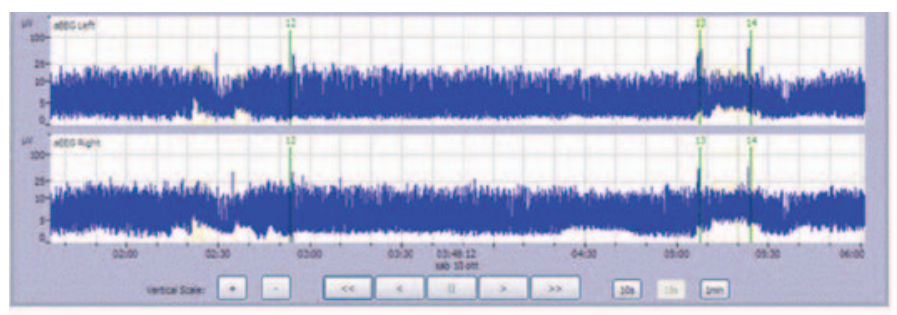

\section{patient 1}

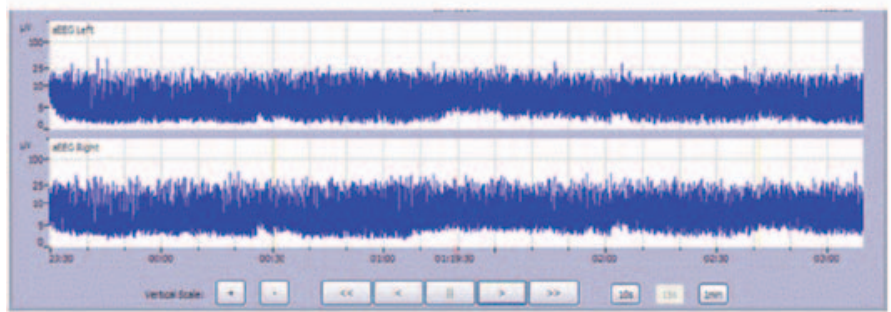

\section{patient 2}

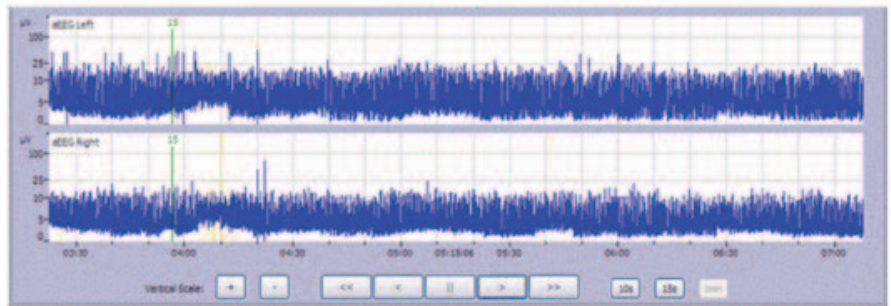

\section{patient 3}

Figure 2. aEEG pattern of patients 1, 2 and 3.

[9]. It is quite surprising to find such percentages, since it is highly unlikely that the number of cases of severe asphyxia is bigger than that of those with moderate asphyxia. These different rates suggest that there might be a subset of infants with a moderate encephalopathy that is properly identified with difficulties or remains undiagnosed with a clinical examination only. We hypothesize that it is simple to identify newborns with severe encephalopathy (corresponding to Sarnat grade 3) but it might not be as easy to diagnose moderate encephalopathy (corresponding to Sarnat grade 2). This difficulty could be due to the fact that clinical signs of HIE may take hours to fully develop [29]. In addition, Sarnat encephalopathy scoring is a well-validated method of outcome prediction only from $24 \mathrm{~h}$ of age [11] and newborns' first neurological examination at their birth hospitals was carried out by noncertified examiners.

One of the more surprising results of our experience was the high percentage $(27.3 \%)$ of newborns with abnormal aEEG in the absence of clinical signs.

Clinical and neuroradiologic follow-up of these infants suggested that asphyxia was definitely more severe than we had thought on the basis of early clinical evaluation. The number of newborns with this contradictory picture is still too small to draw significant conclusions, although the presence of ambiguous signs at the boundary between mild and moderate encephalopathy in a significant percentage of newborns with perinatal asphyxia had already been observed in previous studies [13]. This ambiguity did not allow to categorize some newborns as Sarnat stage 1 or 2, but this finding was relevant because a quarter of these newborns (all having an abnormal early aEEG) progressed to moderate encephalopathy and had an abnormal short-term outcome [13]. Nowadays, uncertainty in clearly discriminating newborns with mild or moderate asphyxia is particularly important as these infants would benefit from treatment with hypothermia.
In clinical trials investigating the efficacy of therapeutic hypothermia, newborns with mild asphyxia (corresponding to a Sarnat grade 1) were excluded from cooling [6-9]. In the CoolCap trial, there was anyway a small proportion of included newborns with mild asphyxia, and neurological outcome was abnormal in a third of them [6]. Both Thoresen et al. [26] and van Handel et al. [31] described a group of newborns classified as Sarnat grade 1 that were cognitively impaired afterwards. An extensive neuropsychological study demonstrated a correlation between mild perinatal asphyxia (Sarnat grade 1) and a worst cognitive outcome [32]. Currently, evidence to propose cooling for neonates with mild asphyxia is insufficient. However, we cannot exclude that within the group of newborns with Sarnat 1 , there is a subgroup exhibiting abnormal aEEG features who could benefit from cooling. It is likely that the systematic early utilization of aEEG for neonates with any degree of perinatal asphyxia might help identifying those, in whom the combined features of early neurologic examination and aEEG will facilitate recognition of term newborns at risk of persistent encephalopathy [13].

The MRI findings in two of the three additional newborns with late neurological disorders and not treated with cooling were consistent with signs of infarction. It is likely that this kind of disorder might explain why these cases were clinically silent initially. In such cases, the aEEG may be an useful tool to ensure a prompt diagnosis of cerebral damage. According to our protocol, these newborns were not cooled, even if currently it is unknown whether cooling after the 6-h treatment window would have been beneficial.

If further studies confirm our hypothesis, a precocious aEEG analysis should be recommended, and a revision of the entry criteria in the current protocol of treatment with hypothermia should be applied. When the metabolic criterion is met, the hypothermia treatment could be reserved to newborns with neurological and/or EEG criteria. 
Declaration of Interest: The authors report no conflicts of interest.

\section{References}

1. Jacobs SE, Tarnow-Mordi WO. Therapeutic hypothermia for newborn infants with hypoxic-ischaemic encephalopathy. J Paediatr Child Health 2010;46:568-576.

2. Parish A, Bhatia J. Hypothermia for hypoxic-ischemic brain injury. J Matern Fetal Neonatal Med 2009;22:719-721.

3. Robertson CM, Finer NN, Grace MG. School performance of survivors of neonatal encephalopathy associated with birth asphyxia at term. J Pediatr 1989;114:753-760.

4. Shankaran S, Woldt E, Koepke T, Bedard MP, Nandyal R. Acute neonatal morbidity and long-term central nervous system sequelae of perinatal asphyxia in term infants. Early Hum Dev 1991;25:135-148.

5. Perlman JM, Wyllie J, Kattwinkel J, Atkins DL, Chameides L, Goldsmith JP, Guinsburg R, et al.; Neonatal Resuscitation Chapter Collaborators. Part 11: Neonatal resuscitation: 2010 International Consensus on Cardiopulmonary Resuscitation and Emergency Cardiovascular Care Science With Treatment Recommendations. Circulation 2010;122:S516-S538.

6. Gluckman PD, Wyatt JS, Azzopardi D, Ballard R, Edwards AD, Ferriero DM, Polin RA, et al. Selective head cooling with mild systemic hypothermia after neonatal encephalopathy: multicentre randomised trial. Lancet 2005;365:663-670.

7. Shankaran S, Laptook AR, Ehrenkranz RA, Tyson JE, McDonald SA, Donovan EF, Fanaroff AA, et al.; National Institute of Child Health and Human Development Neonatal Research Network. Whole-body hypothermia for neonates with hypoxic-ischemic encephalopathy. $\mathrm{N}$ Engl J Med 2005;353:1574-1584.

8. Eicher DJ, Wagner CL, Katikaneni LP, Hulsey TC, Bass WT, Kaufman DA, Horgan MJ, et al. Moderate hypothermia in neonatal encephalopathy: efficacy outcomes. Pediatr Neurol 2005;32:11-17.

9. Azzopardi DV, Strohm B, Edwards AD, Dyet L, Halliday HL, Juszczak E, Kapellou O, et al.; TOBY Study Group. Moderate hypothermia to treat perinatal asphyxial encephalopathy. N Engl J Med 2009;361:1349-1358.

10. Azzopardi D, Guarino I, Brayshaw C, Cowan F, Price-Williams D, Edwards AD, Acolet D. Prediction of neurological outcome after birth asphyxia from early continuous two-channel electroencephalography. Early Hum Dev 1999;55:113-123.

11. Sarnat HB, Sarnat MS. Neonatal encephalopathy following fetal distress. A clinical and electroencephalographic study. Arch Neurol 1976;33:696-705.

12. Simbruner G, Mittal RA, Rohlmann F, Muche R; neo.nEURO.network Trial Participants. Systemic hypothermia after neonatal encephalopathy: outcomes of neo.nEURO.network RCT. Pediatrics 2010;126:e771-e778.

13. Shalak LF, Laptook AR, Velaphi SC, Perlman JM. Amplitude-integrated electroencephalography coupled with an early neurologic examination enhances prediction of term infants at risk for persistent encephalopathy. Pediatrics 2003;111:351-357.

14. Filippi L, Poggi C, la Marca G, Furlanetto S, Fiorini P, Cavallaro G, Plantulli A, et al. Oral topiramate in neonates with hypoxic ischemic encephalopathy treated with hypothermia: a safety study. J Pediatr 2010;157:361-366.

15. Safety and Efficacy of Topiramate in Neonates With Hypoxic Ischemic Encephalopathy Treated With Hypothermia (NeoNATI). NCT01241019. Available at: http://www. clinicaltrials.gov

16. al Naqeeb N, Edwards AD, Cowan FM, Azzopardi D. Assessment of neonatal encephalopathy by amplitude-integrated electroencephalography. Pediatrics 1999;103:1263-1271.
17. Prechtl HF, Einspieler C, Cioni G, Bos AF, Ferrari F, Sontheimer D An early marker for neurological deficits after perinatal brain lesions. Lancet 1997;349:1361-1363.

18. Thompson CM, Puterman AS, Linley LL, Hann FM, van der Elst CW, Molteno CD, Malan AF. The value of a scoring system for hypoxic ischaemic encephalopathy in predicting neurodevelopmental outcome. Acta Paediatr 1997;86:757-761

19. Gunn AJ, Hoehn T, Hansmann G, Bührer C, Simbruner G, Yager J, Levene M, et al. Hypothermia: an evolving treatment for neonatal hypoxic ischemic encephalopathy. Pediatrics 2008;121:648-9; author reply 649.

20. Gunn AJ, Wyatt JS, Whitelaw A, Barks J, Azzopardi D, Ballard R, Edwards AD, et al.; CoolCap Study Group. Therapeutic hypothermia changes the prognostic value of clinical evaluation of neonatal encephalopathy. J Pediatr 2008;152:55-58.

21. Wilkinson DJ. Cool heads: ethical issues associated with therapeutic hypothermia for newborns. Acta Paediatr 2009;98:217-220.

22. Hallberg B, Grossmann K, Bartocci M, Blennow M. The prognostic value of early aEEG in asphyxiated infants undergoing systemic hypothermia treatment. Acta Paediatr 2010; 99:531-536

23. Toet MC, van der Meij W, de Vries LS, Uiterwaal CS, van Huffelen KC. Comparison between simultaneously recorded amplitude integrated electroencephalogram (cerebral function monitor) and standard electroencephalogram in neonates. Pediatrics 2002;109: 772-779.

24. Hellström-Westas L, Rosén I, Svenningsen NW. Predictive value of early continuous amplitude integrated EEG recordings on outcome after severe birth asphyxia in full term infants. Arch Dis Child Fetal Neonatal Ed 1995;72:F34-F38.

25. Spitzmiller RE, Phillips T, Meinzen-Derr J, Hoath SB. Amplitudeintegrated EEG is useful in predicting neurodevelopmental outcome in full-term infants with hypoxic-ischemic encephalopathy: a metaanalysis. J Child Neurol 2007;22:1069-1078.

26. Thoresen M, Hellström-Westas L, Liu X, de Vries LS. Effect of hypothermia on amplitude-integrated electroencephalogram in infants with asphyxia. Pediatrics 2010;126:e131-e139.

27. Horan M, Azzopardi D, Edwards AD, Firmin RK, Field D. Lack of influence of mild hypothermia on amplitude integratedelectroencephalography in neonates receiving extracorporeal membrane oxygenation. Early Hum Dev 2007;83:69-75.

28. Thoresen M. Hypothermia after perinatal asphyxia: selection for treatment and cooling protocol. J Pediatr 2011;158:e45-e49.

29. Sarkar S, Barks JD, Donn SM. Should amplitude-integrated electroencephalography be used to identify infants suitable for hypothermic neuroprotection? J Perinatol 2008;28:117-122.

30. Quigg M, Leiner D. Engineering aspects of the quantified amplitudeintegrated electroencephalogram in neonatal cerebral monitoring. J Clin Neurophysiol 2009;26:145-149.

31. van Handel M, Swaab H, de Vries LS, Jongmans MJ. Long-term cognitive and behavioral consequences of neonatal encephalopathy following perinatal asphyxia: a review. Eur J Pediatr 2007; 166:645-654.

32. de Vries LS, Jongmans MJ. Long-term outcome after neonatal hypoxic-ischaemic encephalopathy. Arch Dis Child Fetal Neonatal Ed 2010;95:F220-F224 